\title{
Vesical imaging reporting and data system (VI-RADS) and impact on identifying depth of invasion with subsequent management in bladder cancer patients: ready for prime time?
}

\author{
Brian S. Wong ${ }^{1}$, Cihan Duran', Stephen B. Williams ${ }^{2}$ \\ ${ }^{1}$ Department of Radiology, The University of Texas Medical Branch, Galveston, TX, USA; ${ }^{2}$ Department of Surgery, Division of Urology, The \\ University of Texas Medical Branch, Galveston, TX, USA \\ Correspondence to: Stephen B. Williams, MD. Division of Urology, The University of Texas Medical Branch, 301 University Blvd, Galveston, TX \\ 77555, USA. Email: stbwilli@utmb.edu.
}

Submitted Mar 31, 2020. Accepted for publication Jun 02, 2020.

doi: $10.21037 /$ tau-20-839

View this article at: http://dx.doi.org/10.21037/tau-20-839

Urothelial carcinoma is the most common histological subtype of bladder cancer, accounting for $90 \%$ in developed countries. Approximately 550,000 new cases of urothelial carcinoma were diagnosed worldwide in 2018 (1). In the United States, 81,400 new cases of bladder cancer will be diagnosed in 2020 and is the sixth most common cancer (2). Despite current treatments, mortality rates remain unaltered over the last 3 decades which may be up to $50-60 \%$ when disease invades the muscle (3). Improved identification of patients at risk of progression and/or muscle-invasion at time of diagnosis are areas of significant research interest.

The classic presentation of urothelial carcinoma is gross hematuria in middle-aged and elderly males. Per the American Urological Association (AUA), patients 35 years and older with asymptomatic microhematuria with no known cause should be evaluated for bladder cancer with cystoscopy (4). Cystoscopy should be performed in patients with risk factors including irritative voiding symptoms, heavy tobacco use, or exposure to known carcinogenic chemicals. If a lesion is identified on flexible cystoscopy, confirmatory transurethral biopsy/resection of visible tumor is performed to assess for depth of invasion. If no definitive lesion is identified, urine cytology may be beneficial in high-risk individual, but has low sensitivity for detecting low-grade tumors (5). Concurrent imaging with retrograde pyelography, computed tomography (CT), magnetic resonance imaging (MRI), or positron emission tomography (PET) may be used for staging the tumor.

Clinical staging is determined by physical examination, imaging, and cystoscopy, but studies have suggested that this method may underestimate the stage of the tumor (6). Pathological staging uses the AJCC TNM staging system $\left(2017,8^{\text {th }}\right.$ ed) to evaluate cystectomy specimens. The most important prognostic determinant is whether the tumor is confined to the bladder $(<\mathrm{T} 2)$ versus invading the bladder muscle (T2) or extends past the bladder into the perivesicular fat ( $>\mathrm{T} 3)$, increasing risk for metastatic spread. Even though the tumor is confined to the bladder, the standard of care for muscle-invasive bladder cancer (MIBC) is radical cystectomy. The majority of bladder cancers identified on cystoscopy are non-muscle invasive bladder cancers (T1) and are managed conservatively with transurethral resection of bladder tumor (TURBT) (1). Determining the presence of muscle invasion in urothelial carcinoma is critical to determining management and may be potentially assessed with advancements in magnetic resonance imaging. Del Giudice et al. proposed using advanced MRI imaging sequences to identify high-risk features in NMIBC that may warrant additional re-TURBT and/or intravesicular chemotherapy (7).

Multiparametric MRI (mpMRI) combines advanced imaging sequences that display anatomic structure and functional information to evaluate the soft tissues of the bladder (8). T2-weighted imaging (T2WI), diffusion weighted imaging (DWI), and dynamic contrast enhancement (DCE) provide valuable information to assess urothelial carcinoma. While the inner layer of urothelium and lamina propria is not visualized, T2WI has high spatial 
Table 1 Sensitivity, specificity, positive predictive value, negative predictive value, and area under the curve of recent studies evaluating VIRADS score $>3$ to predict detrusor muscle invasion in urothelial carcinoma

\begin{tabular}{|c|c|c|c|c|c|c|c|}
\hline Author & Type & Sample size & Sensitivity (\%) & Specificity (\%) & PPV (\%) & NPV (\%) & $A \cup C$ \\
\hline Del Giudice (7) & Prospective & 231 & 91.9 & 91.1 & 77.5 & 97.1 & 0.94 \\
\hline Wang (12) & Retrospective & 340 & 87.1 & 96.5 & 87.1 & 96.5 & 0.94 \\
\hline Kim (13) & Retrospective & 339 & 94.6 & 43.9 & 51.6 & 63.7 & 0.64 \\
\hline
\end{tabular}

PPV, positive predictive value; NPV, negative predictive value; AUC, area under the curve.

resolution that is useful to evaluate the structural integrity of the muscularis propria (detrusor muscle) to differentiate between NMIBC and MIBC. The detrusor muscle appears as a low signal intensity line on T2WI along the bladder wall with intermediate signal intensity on DWI, but no early enhancement on DCE (9). DWI can identify regions of diffusion restriction, which characteristically is seen in urothelial carcinoma; this tumor appears hyperintense on DWI with corresponding hypointensity on the apparent diffusion coefficient (ADC) map. The tumor stalk and inner layer are hypointense on DWI, but show characteristic early enhancement on DCE imaging. Intravesical masses with high signal on DWI, low signal on ADC, and early contrast enhancement on DCE should be assessed and evaluated individually.

The Vesical Imaging Reporting and Data System (VIRADS) is a standardized reporting system that uses T2WI, DWI, and DCE to predict the probability of clinically significant bladder cancer that was proposed in May 2018 by Panabienco et al. (10). The system assigns scores of 1-5 in three categories: structural (SC), contrast enhanced (CE), and diffusion weighted (DW). SC relies on the inherent low T2 properties of muscle to assess the integrity of the detrusor muscle from the intermediate signal intensity of tumor. Tumor size, morphology, and degree of invasion are assessed to determine the score for SC. CE assesses the integrity of the muscularis propria by exploiting its lack of early contrast enhancement. The presence of a hypointense line under the early-enhancing tumor and inner layer corresponds to an intact muscularis propria with a low CE score. On DW, the presence of an intact intermediate signal intensity muscularis propria under the hyperintense tumor corresponds to a low DW score. The system assigns a final score of 1 to 5 that corresponds to highly unlikely [1], unlikely [2], equivocal [3], likely [VIRADS 4], and very likely [5] muscle invasion. Early studies with the VIRADS system have shown a high interobserver agreement and diagnostic accuracy $(9,11)$.

The current article assesses the clinical impact of VIRADS on management of high-risk NMIBC patients for repeat TURBT. The authors first endpoint was to assess the ability of VIRADS to discriminate between NMIBC from MIBC. mpMRI was offered to all eligible patients with urothelial carcinoma prior to undergoing TURBT and the VIRADS score was compared to pathological grade of tumor samples during TURBT. A cutoff of $\geq 3$ was used to define MIBC. mpMRI has a good sensitivity and specificity of $92 \%$ and $91 \%$ to differentiate NMIBC from MIBC (8). The secondary endpoint of the study was to assess VIRADS score as a predictor of re-TURBT pathology in patients with high-risk NMIBC. mpMRI has a sensitivity and specificity of $85 \%$ and $94 \%$ to identify muscle invasion in high-risk patients undergoing re-TURBT (8). Retrospective studies have found similar results for the ability of mpMRI to identify MIBC with sensitivity and specificity of $87 \%$ and $96.5 \%$ (12). Raising the cutoff of VIRADS to $\geq 4$ may increase the specificity and diagnostic accuracy of mpMRI to identify MIBC, at the expense of decreasing sensitivity (13) (Table 1).

While the results of using a standardized system with mpMRI are promising, there are a number of considerations before VIRADS can be used in widespread clinical practice. The first consideration is the high cost associated with mpMRI, which may lead to a delay in treatment. While mpMRI may be potentially useful in guiding therapy, treatment should not be delayed due to the high costs associated with disease progression, especially in high-risk NMIBC patients. High resolution scans of $\geq 3$-Tesla with a standardized protocol are necessary, which were addressed in this prospective, blinded study. In addition to the protocol, the technique must be standardized with a specified endpoint to bladder distention and pharmacotherapy to address visceral motion artifact. Additional artifact may be caused by recent TURBT or intravesical therapy, resulting in fibrosis 
or inflammation that mimics low T2-signal of the detrusor muscle. DWI and DCE sequences can improve the ability for mpMRI to differentiate between post-TURBT changes and residual tumor, increasing the diagnostic accuracy.

The use of mpMRI with a novel standardized reporting system has many potential clinical applications. The high diagnostic accuracy and non-invasive nature make it a candidate for staging individuals with urothelial carcinoma and identify high-risk individuals who may need reTURBT or proceed to radical cystectomy. mpMRI can further stage patients by assessing tumor spread to lymph nodes, bones, and upper urinary tract, which may be key to guiding management (14). While cystoscopy and TURBT are the gold standard for diagnosis, MRI is an adjunct that may be beneficial in a variety of scenarios. Future multicenter studies are warranted to determine the optimal timing, VIRADS score, and patient populations that may benefit from mpMRI.

\section{Acknowledgments}

Funding: None.

\section{Footnote}

Provenance and Peer Review: This article was commissioned by the editorial office, Translational Andrology and Urology. The article did not undergo external peer review.

Conflicts of Interest: All authors have completed the ICMJE uniform disclosure form (available at http://dx.doi. org/10.21037/tau-20-839). The authors have no conflicts of interest to declare.

Ethical Statement: The authors are accountable for all aspects of the work in ensuring that questions related to the accuracy or integrity of any part of the work are appropriately investigated and resolved.

Open Access Statement: This is an Open Access article distributed in accordance with the Creative Commons Attribution-NonCommercial-NoDerivs 4.0 International License (CC BY-NC-ND 4.0), which permits the noncommercial replication and distribution of the article with the strict proviso that no changes or edits are made and the original work is properly cited (including links to both the formal publication through the relevant DOI and the license). See: https://creativecommons.org/licenses/by-nc-nd/4.0/.

\section{References}

1. Bray F, Ferlay J, Soerjomataram I, et al. Global cancer statistics 2018: GLOBOCAN estimates incidence and mortality worldwide for 36 cancers in 185 countries. CA Cancer J Clin 2018;68:394-424.

2. Siegel RL, Miller KD, Jemal A. Cancer statistics, 2020. CA Cancer J Clin 2020;70:7-30.

3. Chamie K, Litwin MS, Bassett JC, et al. Recurrence of high-risk bladder cancer: a population-based analysis. Cancer 2013;119:3219-27.

4. Davis R, Jones JS, Barocas DA, et al. Diagnosis, evaluation and follow-up of asymptomatic microhematuria (AMH) in adults: AUA guideline. J Urol 2012;188:2473-81.

5. Yafi FA, Brimo F, Steinberg J, et al. Prospective analysis of sensitivity and specificity of urinary cytology and other urinary biomarkers for bladder cancer. Urol Oncol 2015;33:66.e25-31.

6. Shariat SF, Palapattu GS, Karakiewicz PI, et al. Discrepancy between clinical and pathologic stage: impact on prognosis after radical cystectomy. Eur Urol 2007;51:137-49.

7. Del Giudice F, Barchetti G, De Berardinis E, et al. Prospective Assessment of Vesical Imaging Reporting and Data System (VI-RADS) and Its Clinical Impact on the Management of High-risk Non-muscle-invasive Bladder Cancer Patients Candidate for Repeated Transurethral Resection. Eur Urol 2020;77:101-9.

8. de Haas RJ, Steyvers MJ, Fütterer JJ. Multiparametric MRI of the bladder: ready for clinical routine? AJR Am J Roentgenol 2014;202:1187-95.

9. Ueno Y, Takeuchi M, Tamada T, et al. Diagnostic Accuracy and Interobserver Agreement for the Vesical Imaging-Reporting and Data System for Muscle-invasive Bladder Cancer: A Multireader Validation Study. Eur Urol 2019;76:54-6.

10. Panebianco V, Narumi Y, Altun E, et al. Multiparametric Magnetic Resonance Imaging for Bladder Cancer: Development of VI-RADS (Vesical Imaging-Reporting And Data System). Eur Urol 2018;74:294-306.

11. Barchetti G, Simone G, Ceravolo I, et al. Multiparametric MRI of the bladder: inter-observer agreement and accuracy with the Vesical Imaging-Reporting and Data System (VI-RADS) at a single reference center. Eur Radiol 2019;29:5498-506.

12. Wang H, Luo C, Zhang F, et al. Multiparametric MRI for Bladder Cancer: Validation of VI-RADS for the Detection of Detrusor Muscle Invasion. Radiology 2019;291:668-74. 
13. Kim SH. Validation of vesical imaging reporting and data system for assessing muscle invasion in bladder tumor. Abdom Radiol (NY) 2020;45:491-8.

Cite this article as: Wong BS, Duran C, Williams SB. Vesical imaging reporting and data system (VI-RADS) and impact on identifying depth of invasion with subsequent management in bladder cancer patients: ready for prime time? Transl Androl Urol 2020;9(6):2467-2470. doi: 10.21037/tau-20-839
14. Caglic I, Panebianco V, Vargas HA, et al. MRI of Bladder Cancer: Local and Nodal Staging. J Magn Reson Imaging 2020;52:649-67. 